\title{
TGF- $\beta 3$ reduces apoptosis in ischemia-induced adipose-derived stem cells by enhancing DNA repair
}

\author{
FAN WU ${ }^{1,2^{*}}$, HAIWEN YE $^{1,2^{*}}$, JUNFENG LIN $^{1,2}$, YAODONG XU $^{1,2}$, ZHUASONG ZHANG $^{1,2}$, \\ HAO XIONG ${ }^{1,2}$, MAOJIN LAING ${ }^{1,2}$, YIQING ZHEN ${ }^{1,2}$ and SUIJUN CHEN ${ }^{1,2}$ \\ ${ }^{1}$ Department of Otorhinolaryngology; ${ }^{2}$ The Guangdong Provincial Key Laboratory of \\ Malignant Tumor Epigenetics and Gene Regulation, Sun Yat-sen Memorial Hospital, \\ Sun Yat-sen University, Guangzhou, Guangdong 510000, P.R. China
}

Received October 23, 2017; Accepted February 16, 2018

DOI: $10.3892 /$ etm.2018.5980

\begin{abstract}
Adipose-derived stem cells (ADSCs) possess good proliferative and differentiative abilities, making then a promising candidate for the treatment of cartilage defects. However, local ischemia often causes apoptosis in ADSCs. Transforming growth factor- $\beta 3$ (TGF- $\beta 3$ ) is often used as a chondrogenic differentiation cytokine whose function in apoptosis is unclear. The aim of the present study was to investigate the role of TGF- $\beta 3$ in ischemia-induced ADSC apoptosis. In the present study, the phenotypes and multipotent differentiation properties of human ADSCs at passage 3 were analyzed using flow cytometry and cytochemical staining. ADSCs were cultured in a serum- and glucose-free medium under hypoxic conditions with or without exogenous TGF- $\beta 3$ treatment. The apoptosis rate was measured using a TUNEL array and Annexin V/propidium iodide staining. The expression of apoptosis-associated proteins was measured using western blotting. The results revealed ADSCs cultured in normal condition have multi-lineage differentiation potential and high levels of cluster of differentiation (CD)29, CD44 and CD105 expression. Furthermore, ADSCs weakly express CD14, CD34 and CD45, with strong clone formation and migration abilities. Serum deprivation under hypoxic conditions resulted in mitochondria-mediated apoptosis in ADSCs, which was attenuated by exogenous TGF- $\beta 3$ treatment via upregulation of poly ADP-ribose polymerase (PARP). The results of the present study indicate that TGF- $\beta 3$ is able to protect ADSCs
\end{abstract}

Correspondence to: Dr Suijun Chen, Department of Otorhinolaryngology, Sun Yat-sen Memorial Hospital, Sun Yat-sen University, 107 Yan Jiang Xi Road, Guangzhou, Guangdong 510000, P.R. China

E-mail: chensuijun1974@163.com

*Contributed equally

Key words: adipose-derive stem cells, ischemia, apoptosis, transforming growth factor- $\beta 3$, poly ADP-ribose polymerase from ischemia-induced apoptosis via PARP-associated DNA damage repair.

\section{Introduction}

The repair of damaged cartilage is clinically challenging as cartilage has a low capacity for self-repair (1). Traditional therapies include autologous tissue replacement and alloplastic implants, which can result in foreign body reactions and local fibrosis (2). Recently, stem-cell-based tissue engineering has come to be regarded as a potential ideal strategy to address this problem (3). Adipose-derived stem cells (ADSCs) are abundant and readily available (4); furthermore, their chondrogenic transformative ability makes them promising seed cells for cartilage repair (5). However, this strategy is limited as ischemia results in low survival rats in transplant sites (6).

Transforming growth factor (TGF)- $\beta 3$ is an important cytokine that serves a role in the induction of chondro-lineage differentiation in ADSCs (7). TGF- $\beta 3$ is structurally and biologically distinct from TGF- $\beta 1$ and TGF- $\beta 2$ (8) and is able to promote cartilage repair and accelerate cartilage differentiation. Associated studies have demonstrated that TGF- $\beta 3$ is cytokine important for the differentiation of ADSCs into chondrocytes (9).

Whether TGF- $\beta 3$ is a pro-apoptotic factor remains controversial (10-12). To the best of our knowledge, the effects of TGF- $\beta 3$ on ischemia-induced apoptosis in ADSCs have not previously been investigated. The aim of the present study was to determine the effects and potential mechanisms of TGF- $\beta 3$ on ischemic-induced death in ADSCs.

\section{Materials and methods}

Culture and identification of human ADSCs. Human ADSCs as passage two were purchased from Cyagen Biosciences, Inc. (Santa Clara, CA, USA) and cultured in complete Dulbecco's modified Eagle's medium (DMEM; Gibco; Thermo Fisher Scientific, Inc., Waltham, MA, USA) containing 10\% fetal bovine serum (FBS; Gibco; Thermo Fisher Scientific, Inc.) in a humidified atmosphere containing $5 \% \mathrm{CO}_{2}$ at $37^{\circ} \mathrm{C}$. The medium was changed every $48 \mathrm{~h}$. Once cells reached $80 \%$ confluence they were passaged by digestion with $0.25 \%$ 
trypsin/EDTA (Gibco; Thermo Fisher Scientific, Inc.) and seeded in $25 \mathrm{~cm}^{2}$ flasks at a density of $1 \times 10^{5}$ cells $/ \mathrm{cm}^{2}$.

Cultured human ADSCs were analyzed using a flow cytometer (BD Biosciences, Franklin Lakes, NJ, USA). Passage 3 ADSCs were digested with $0.25 \%$ trypsin/EDTA, washed in PBS and resuspended in PBS at $5 \times 10^{6}$ cells $/ \mathrm{ml}$. After the cells were blocked with $10 \%$ goat serum (Wuhan Boster Biological Technology, Ltd., Wuhan, China) at room temperature for $30 \mathrm{~min}$, they were subsequently incubated for $15 \mathrm{~min}$ at $4^{\circ} \mathrm{C}$ in the dark with the following antibodies: Anti-cluster of differentiation (CD)29-fluorescein isothiocyanate (FITC), anti-CD44-FITC (cat. no. 560977), anti-CD105-phycoethrin (PE; cat. no. 560839), anti-CD14-PE (cat. no. 557154), anti-CD34-FITC (cat. no. 560942) and anti-CD45-FITC (cat. no. 555482; all BD Biosciences). All the above antibodies were at a dilution of 1:500. Cells were washed twice with PBS and analyzed using CellQuest software (version 0.9.13 alpha; BD Biosciences).

Differentiation properties of human ADSCs. To assess osteogenic differentiation, ADSCs were seeded $\left(5 \times 10^{3}\right.$ cells $\left./ \mathrm{cm}^{2}\right)$ in 6-wells plates containing complete DMEM. Cells were cultured for $48 \mathrm{~h}$, following which the medium was changed to osteogenic induction medium from the Osteogenesis Differentiation kit (Cyagen Biosciences, Inc.) and cells were cultured for a further 20 days. The cells were subsequently stained with a $1 \%$ Alizarin Red S solution at room temperature for $15 \mathrm{~min}$.

A total of $6 \mu$ 1 ADSCs $\left(\sim 1 \times 10^{7}\right.$ cells $)$ were seeded in the bottom of a centrifuge tube with $5 \mathrm{ml}$ DMEM containing $10 \%$ FBS. Cells were cultured for $48 \mathrm{~h}$ and the medium was changed to chondrogenic induction medium using a Chondrogenesis Differentiation kit (Cyagen Biosciences, Inc.). The medium was changed every 3 days. Chondro-lineage differentiation was induced for 15 days and the cells stained with $1 \%$ Alcian Blue at room temperature for $15 \mathrm{~min}$.

To induce adipogenic differentiation, ADSCs were seeded on 6 -wells plates at $1 \times 10^{4}$ cells $/ \mathrm{cm}^{2}$ in complete DMEM. Following $24 \mathrm{~h}$ of culture, the medium was changed to adipogenic inductive medium using an Adipogenesis Differentiation kit (Cyagen Biosciences, Inc.) and the cells were cultured for 15 days. Oil red $\mathrm{O}$ staining $(0.3 \%)$ was used at room temperature for $15 \mathrm{~min}$ to observe oil droplets in the induced cells.

Colony-forming cell assay. ADSCs were seeded in 6-well plates at a density of $1 \times 10^{3}$ cells/well and cultured for 14 days. Colony formation was observed using light microscopy (Olympus Corp., Tokyo, Japan) at a magnification of x100. After staining with $1 \%$ crystal violet at room temperature for 15 min and washing with PBS, the number of colony-forming units (CFU) was counted and the colony-forming rate was calculated as follows: no. CFUs/no. starting cells/well x100.

Cell migration assay. Uniform wound lines were introduced into ADSC cultures using a culture insert (Ibidi, Munich, Germany). The inserts were placed in individual wells of a 6-well plate and removed following cell attachment. The gaps were washed with PBS and $2 \mathrm{ml}$ DMEM was added to each well. Cell migration into the wound area was recorded using an inverted microscope (Olympus Corp.) at 0, 24, 48 and $72 \mathrm{~h}$.
OSD treatment. ADSCs were seeded at a density of $2 \times 10^{4}$ cells $/ \mathrm{cm}^{2}$ on glass coverslips for the TUNEL assay and in 6-well plates for western blotting and the Annexin V/propidium iodide (PI) assay. A pilot study revealed that $10 \mathrm{ng} / \mathrm{ml}$ of TGF- $\beta 3$ was sufficient to induce ADSC differentiation into chondrocytes (data not shown), and so this concentration was selected for use in the following experiments. To mimic ischemic conditions and analyze the effects of every component of these conditions, cells were divided into the oxygen-deprivation group (OD) and oxygen and serum deprivation group (OSD). The OD group was cultured in DMEM containing 10\% FBS, and the OSD group was cultured in serum-free DMEM. To analyze the effect of TGF- $\beta 3$ on the OSD group, the culture medium was replaced with serum-free DMEM with or without $10 \mathrm{ng} / \mathrm{ml}$ TGF- $\beta 3$ (the OSD+TGF- $\beta 3$ group). Cells were cultured under hypoxic conditions in an atmosphere containing 95\% $\mathrm{N}_{2}$ and $5 \% \mathrm{CO}_{2}$ at $37^{\circ} \mathrm{C}$ for $48 \mathrm{~h}$ as previously reported (6). ADSCs cultured in complete DMEM in a $5 \% \mathrm{CO}_{2}$ humidified atmosphere at $37^{\circ} \mathrm{C}$ served as the negative control (NC) group.

Flow cytometric Annexin V-FITC/PI assay of ADSCs. An Annexin V/PI Apoptosis kit (Thermo Fisher Scientific, Inc.) was used to assess the rate of apoptosis in ADSCs following ischemia according to the manufacturer's protocol. Briefly, ADSCs rom each group were digested with $0.25 \%$ trypsin/EDTA. Cells were washed with PBS, re-suspended in binding buffer and incubated for $15 \mathrm{~min}$ at room temperature in the dark with $5 \mu \mathrm{l}$ Annexin V-FITC and $10 \mu \mathrm{l}$ PI. The Annexin V-FITC and PI-labeled cells were analyzed using a flow cytometer (BD Biosciences). Using flow cytometry and CellQuest software, dot plots of PI on the y-axis against Annexin V-FITC on the $\mathrm{x}$-axis were used to distinguish viable cells, early apoptotic cells and late apoptotic or necrotic cells.

TUNEL assay of the ischemic ADSCs. A TUNEL assay was performed to measure double stranded DNA cleavage using one-step TUNEL kit (Beyotime Institute of Biotechnology, Haimen, China). Cells were fixed with $10 \%$ formalin at room temperature for $1 \mathrm{~h}$ and groups of slips were incubated in permeabilisation solution (1\% Triton X-100 in PBS, freshly prepared) for $10 \mathrm{~min}$ on ice. The slides were washed three times with PBS and incubated with TUNEL working solution in a humidified chamber at $37^{\circ} \mathrm{C}$ for $1 \mathrm{~h}$. Samples were counterstained with DAPI in room temperature for $5 \mathrm{~min}$. following the mounting of the coverslips with glycerin (Beyotime Institute of Biotechnology), the stained coverslips were examined under a fluorescence microscope (Olympus Corp.). TUNEL positive cells in 4 random fields were counted and analyzed with GraphPad Prism software (version 5.0; GraphPad Software, Inc., La Jolla, CA, USA) for each group (magnification, x200).

PARP gene silencing by siRNA.PARP was inhibited using small interfering RNA (siRNA). Cells $\left(2.5 \times 10^{5}\right.$ cells/well) were seeded in 6-well plates and transfected with $20 \mathrm{nM}$ PARP-targeting si-RNA (5'-CCAACAGAAGTACGTGCAA-3') or control si-RNA (5'-CCCTCTGCACTAATCTGAA-3'; both Santa Cruz Biotechnology, Inc., Dallas, TX, USA) using Lipofectamine ${ }^{\circledR}$ 3000 (Thermo Fisher Scientific, Inc.) according to the manufacturer's protocol and cultured for $72 \mathrm{~h}$ at $37^{\circ} \mathrm{C}$ in a $5 \% \mathrm{CO}_{2}$ 
A

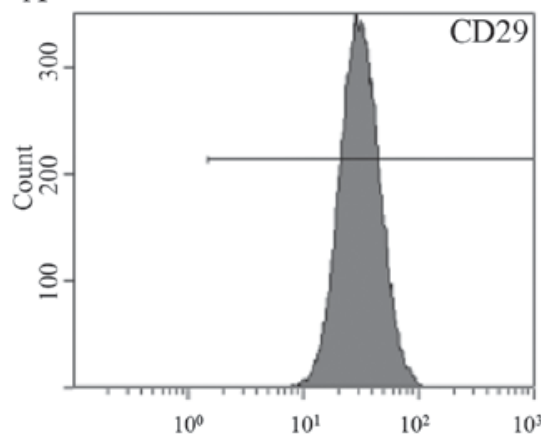

D

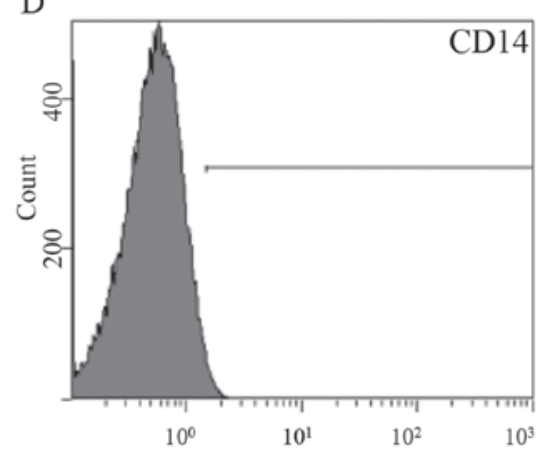

B

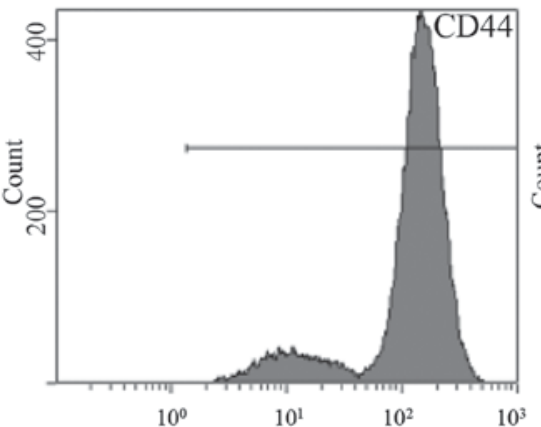

E

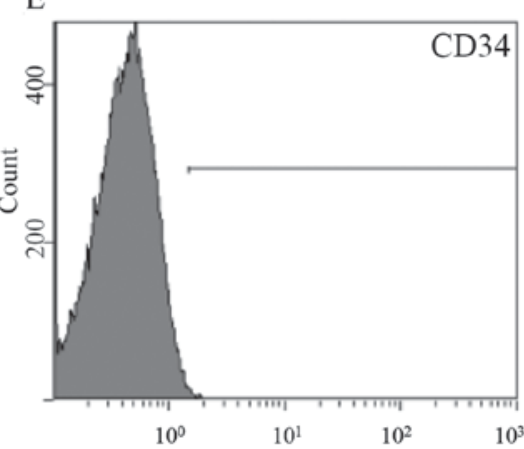

C

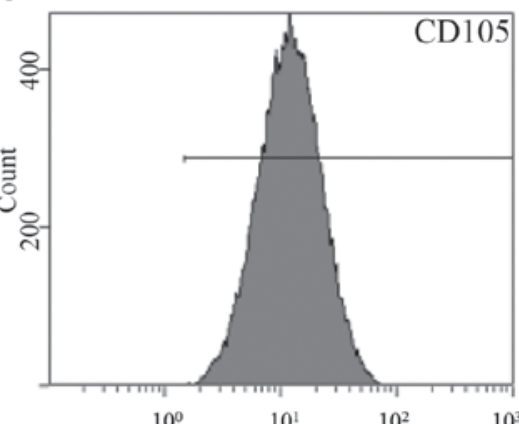

$\mathrm{F}$

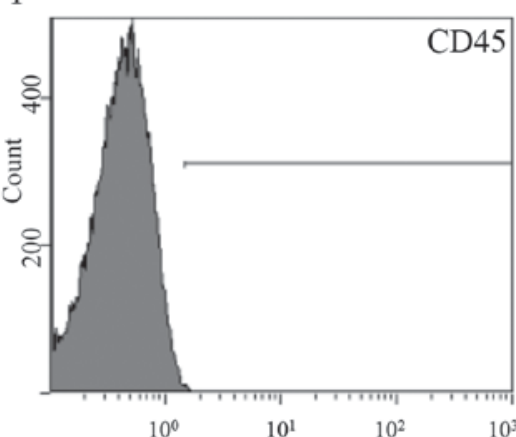

Figure 1. Flow cytometric analysis of stem cell markers (A) CD29, (B) CD44, (C) CD105, (D) CD14, (E) CD34 and (F) CD45 in adipose-derived stem cells. $\mathrm{CD}$, cluster of differentiation.

incubator. PARP expression was subsequently measured by western blotting as described below. Transfected ADSCs were cultured under OSD+TGF- $\beta 3$ conditions and analyzed using western blotting (as described below) and Annexin V/PI staining (as described above).

Western blotting. ADSCs were homogenized in radioimmunoprecipitation assay buffer (Sigma Aldrich; Merck KGaA, Darmstadt, Germany) on ice for $30 \mathrm{~min}$. Proteins were quantified using a bicinchoninic acid assay kit (Beyotime Institute of Biotechnology) and equal amounts of proteins (20 $\mu \mathrm{l} /$ lane) were separated using 10\% SDS-PAGE. Proteins were transferred onto polyvinylidene fluoride membranes and blocked in 5\% non-fat milk in TBS-0.1\% Tween-20 for $1 \mathrm{~h}$ at room temperature. The membranes were subsequently incubated overnight at $4{ }^{\circ} \mathrm{C}$ with primary antibodies against caspase 3 (cat. no. \#9662; 1:500), activated caspase 3 (cat. no. \#9664; 1:1,000), PARP (cat. no.\#9532; 1:1,000), activated PARP (cat. no. \#5625; 1:1,000), B-cell lymphoma 2 (Bcl-2; cat. no. \#4223; 1:1,000), Bcl-2-associated X protein (Bax; cat. no. \#5023; 1:1,000), activated caspase 9 (cat. no. \#9505; 1:1,000) and GAPDH (cat. no. \#5174; 1:10,000). Following washing with TBST, membranes were incubated with horseradish peroxidase-conjugated anti-rabbit secondary antibodies (cat. no. \#7074; 1:3,000) for $1 \mathrm{~h}$ at room temperature. All the above antibodies were purchased from Cell Signaling Technology, Inc. (Danvers, MA, USA). Bands were visualized using an enhanced chemiluminescence kit (EMD Millipore, Billerica, MA, USA). Images of the bands were captured using a Bio-Rad Gel Doc XR documentation system (Bio-Rad Laboratories, Inc., Hercules, CA, USA). Relative protein expression was determined using densitometry and standardized to GAPDH levels using ImageJ software (version 2.1.7.4; National Institutes of Health, Bethesda, MA, USA).

Statistical analysis. Data are presented as the mean \pm or + standard error of the mean. All experiments were performed six times to provide sufficient data. Differences between experimental conditions and controls were analyzed using one-way analysis of variance followed by Tukey's post hoc test and GraphPad Prism software. $\mathrm{P}<0.05$ was considered to indicate a statistically significant difference.

\section{Results}

Identification, differentiation potential and stem cell properties analysis of ADSCs. Flow cytometry revealed that ADSCs expressed stem cell-associated markers CD29, CD44, CD105 and CD90 (Fig. 1A-C). However, expression of the mononuclear phagocyte marker CD14, hematopoietic cell marker CD34 and leukocyte marker CD45 was not observed in ADSCs (Fig. 1D-F).

Cell differentiation was induced using 3-lineage differentiation kits. ADSCs differentiated into osteoblasts (Fig. 2A; Alizarin Red staining), chondrocytes (Fig. 2B; Alcian Blue staining) and adipocytes as confirmed by (Fig. 2C; Oil Red O staining). These results demonstrate that ADSCs are capable of multi-lineage differentiation.

The results of cell migration assays revealed that the migration rate of ADSCs was $36.2 \pm 4.0 \%$ in $24 \mathrm{~h}, 69.0 \pm 5.5 \%$ in $48 \mathrm{~h}$ and $89.6 \pm 7.2 \%$ in $72 \mathrm{~h}$ (Fig. 2D-E). Colony formation was measured following 6 days of culture. Colony formation was $37 \pm 3.5 \%$, demonstrating that cultured ADSCs have the capacity for self-renewal (Fig. 2F-G). 

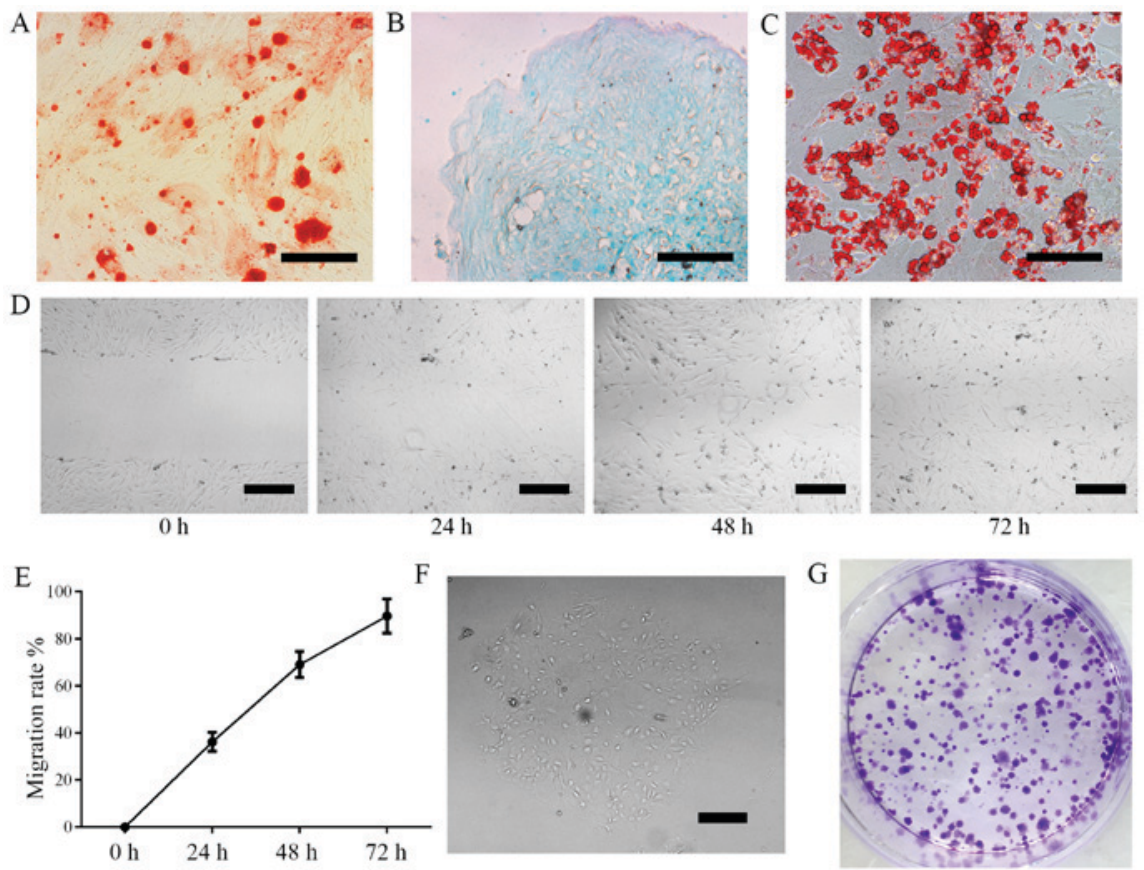

Figure 2. Adipose-derived stem cells were induced to differentiate into (A) osteoblasts, (B) chondrocytes and (C) adipocytes using lineage-specific differentiation kits. (D) A migration assay was performed at different time points and (E) the results were analyzed. (F) Colony-forming cells observed under a light microscope. (G) Colony-forming cell assay stained with crystal violet. Scale bar=20 $\mu \mathrm{m}$.
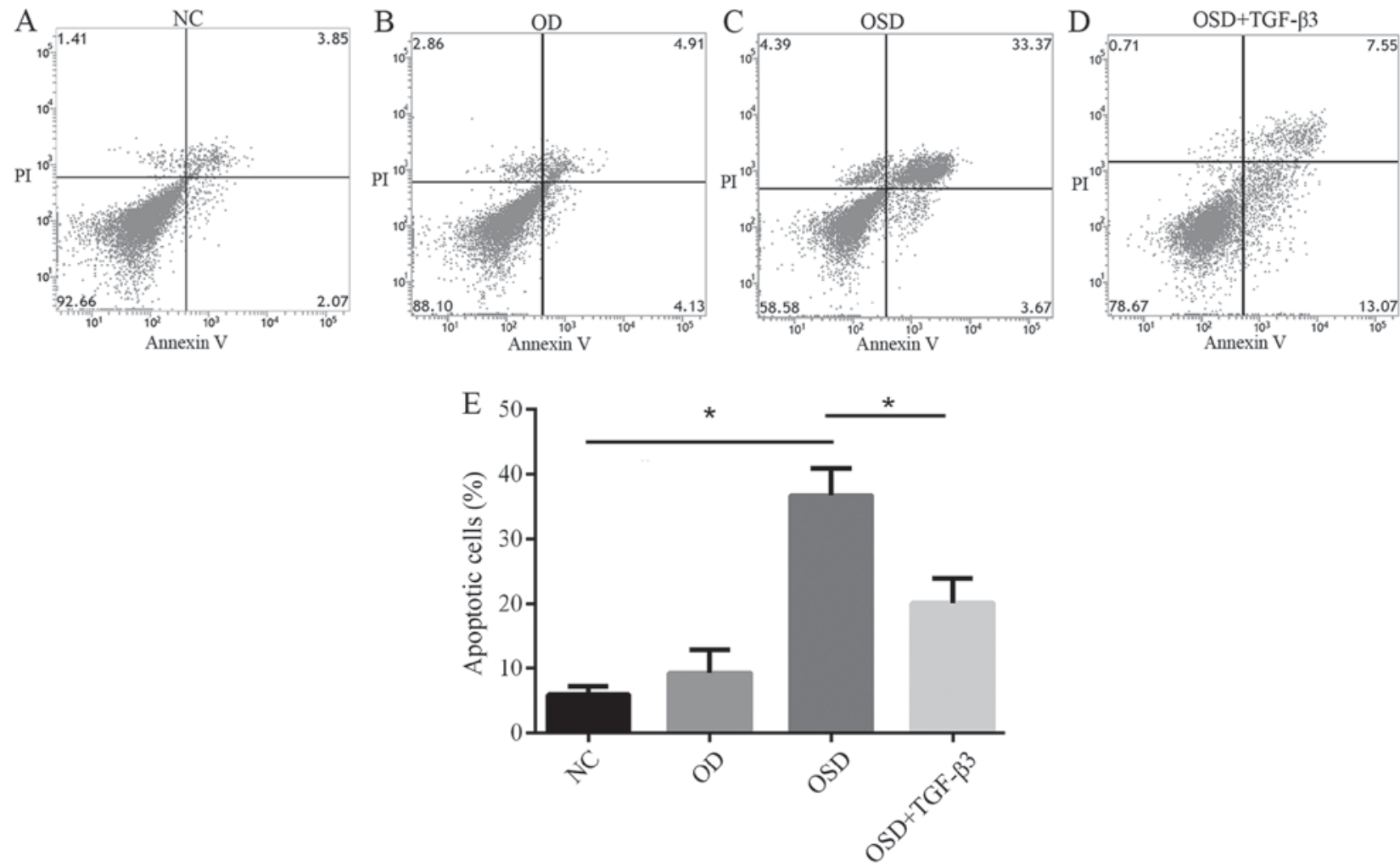

Figure 3. Flow cytometric analysis of apoptosis in the (A) NC, (B) OD, (C) OSD and (D) OSD + TGF- $\beta 3$ groups. The lower left quadrant represents normal cells, lower right quadrant represents cells in the early stages of apoptosis and the upper right quadrant represents cells in late apoptosis. (E) The number of apoptotic cells of each group in the NC, OD, OSD and OSD + TGF- $\beta 3$ groups. " $\mathrm{P}<0.05$. NC, negative control; OD, oxygen deprivation; OSD, oxygen and serum deprivation; TGF, transforming growth factor; PI, propidium iodide.

ADSC apoptosis is induced by OSD and alleviated by TGF- $\beta 3$ treatment. To investigate the effect of TGF- $\beta 3$ on ischemia-associated apoptosis of ADSCs in vitro, ADSCs were exposed to OD or OSD conditions for $48 \mathrm{~h}$, with or without exogenous TGF- $\beta 3$. Apoptosis was assessed using an Annexin V/PI Apoptosis kit (Fig. 3). The percentage of apoptotic ADSCs 

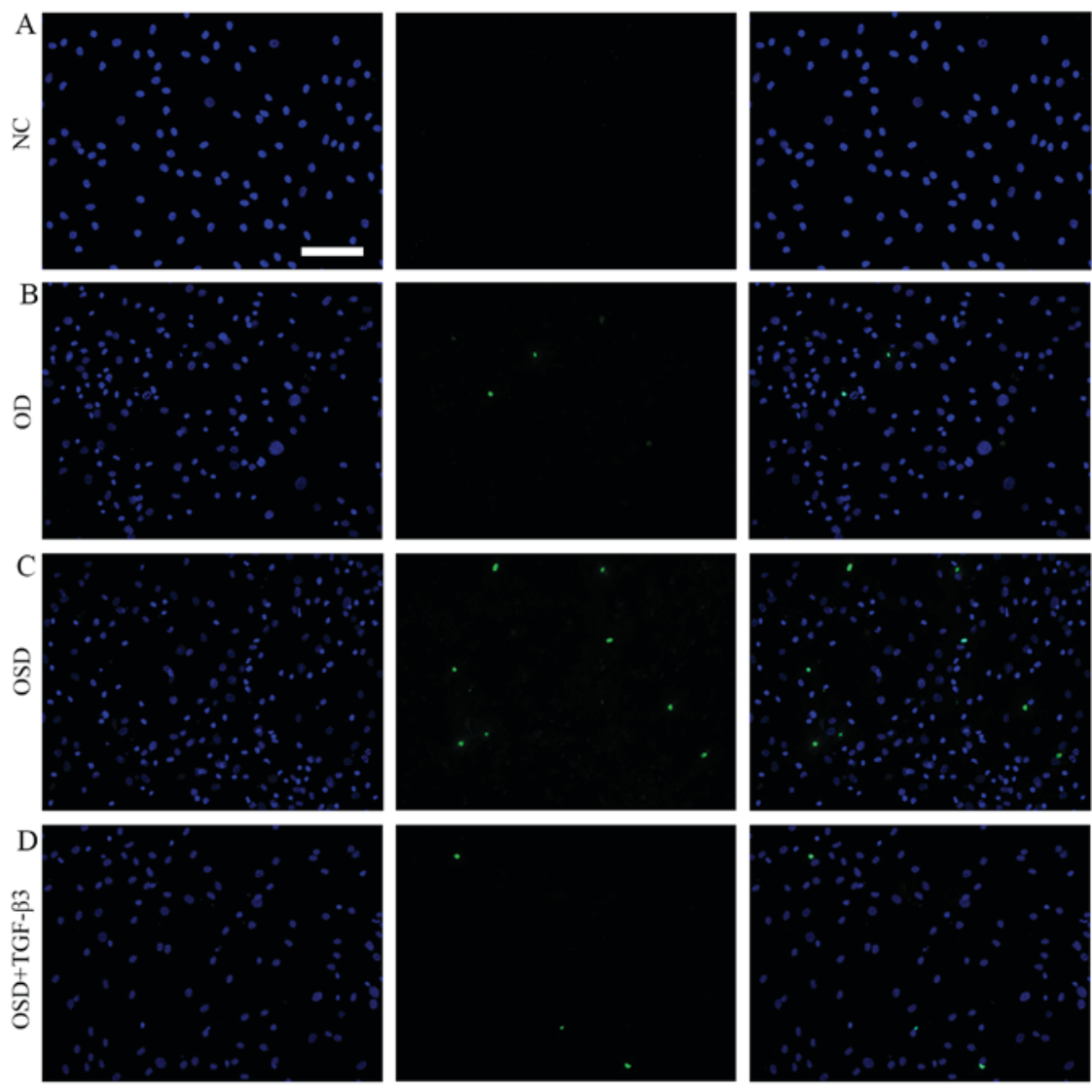

DAP

TUNEL

Merge

Figure 4. DAPI and TUNEL co-staining was used to assess apoptosis in the (A) NC, (B) OD, (C) OSD and (D) OSD + TGF- $\beta 3$ groups. Scale bar=50 $\mu \mathrm{m}$. NC, negative control; OD, oxygen deprivation; OSD, oxygen and serum deprivation; TGF, transforming growth factor.

in the NC, OSD, OD and OSD+TGF- $\beta 3$ groups were $5.93 \pm 1.30$, $9.22 \pm 3.63,36.72 \pm 4.15$ and $20.11 \pm 3.78 \%$, respectively (Fig. 3E). These results suggest that OD alone did not result in apoptosis in ADSCs, which is consistent with previous research (13). A previous study reported that low oxygen conditions may improve ADSC proliferation ability (14). However, serum deprivation under hypoxic conditions effectively induced apoptosis (Fig. 3E). Based on these results, it was hypothesized that different components of ischemia exert their own effects on ADSCs Adding TGF- $\beta 3$ to the culture medium significantly reduced the percentage of apoptotic cells. These results demonstrate that TGF- $\beta 3$ may reverse OSD-induced apoptosis by preventing cells from entering the later stages of apoptosis.

To further investigate apoptosis in ADSCs, TUNEL staining was performed (Fig. 4). Following treatment with OSD, nuclear condensation and cell shrinkage were observed. The number of TUNEL-positive cells in the OSD group was significantly increased compared with the NC group (Fig. 5). The addition of TGF- $\beta 3$ significantly reduced the number of TUNEL-positive cells compared with the OSD group (Fig. 5). TUNEL positive cells represent cells that underwent programmed breaks of DNA double strands. Together, these results suggest that exogenous TGF- $\beta 3$ is able to improve OSD-resistance in ADSCs.

TGF- $\beta 3$ attenuates OSD-induced apoptosis in ADSCs. To analyze the mechanism by which TGF- $\beta 3$ prevents OSD-induced apoptosis in ADSCs, changes in the

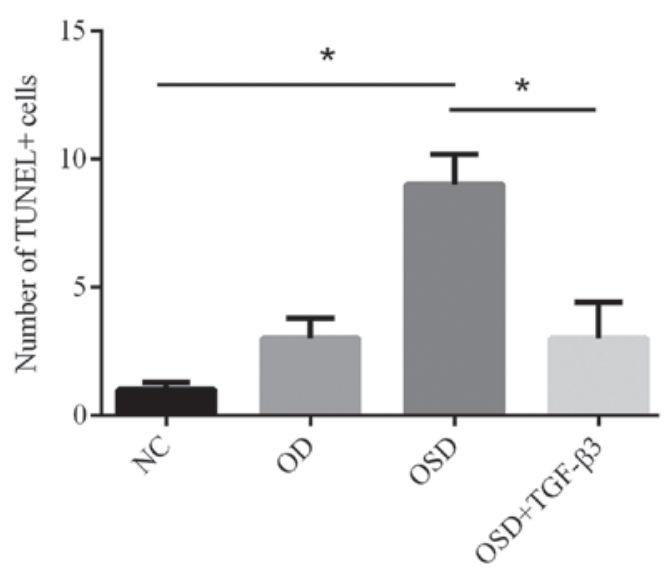

Figure 5. TUNEL-positive cells of each group in the NC, OD, OSD and OSD + TGF- $\beta 3$ groups. ${ }^{*} \mathrm{P}<0.05$. NC, negative control; OD, oxygen deprivation; OSD, oxygen and serum deprivation; TGF, transforming growth factor.

expression of apoptotic genes was assessed using western blotting (Fig. 6). Caspase 3 is a key effector molecule in the downstream signaling pathways associated with apoptosis, which, together with other effector caspases, including caspase 7 and caspase 6, orchestrates the dismantling of diverse cell structures via cleavage of specific substrates (15). No significant difference in caspase 3 expression levels was observed between the NC and OD groups (Fig. 3B). However, caspase 3 was significantly downregulated and activated caspase 3 
A
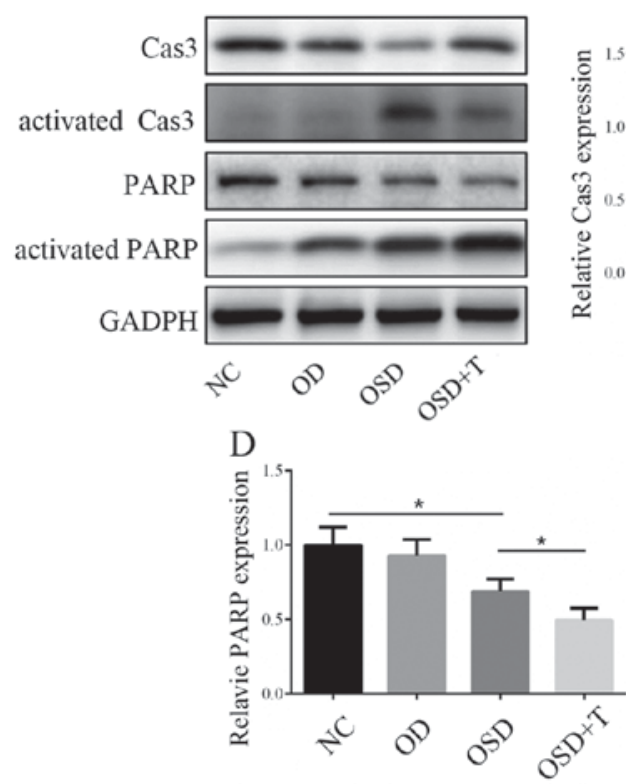

B

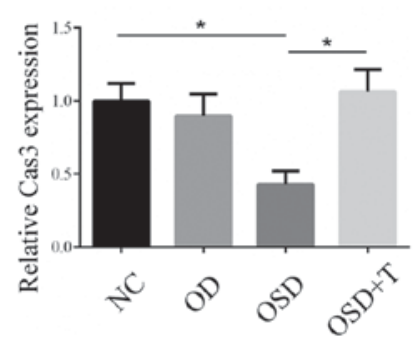

C

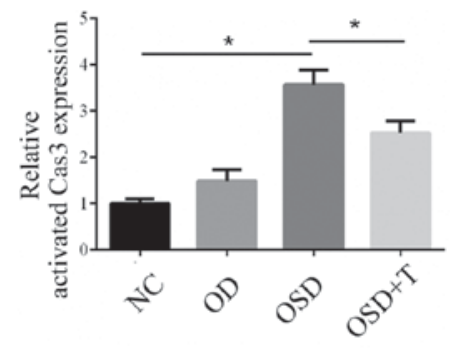

E

F

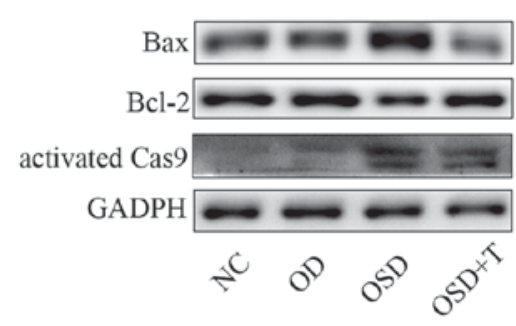

G

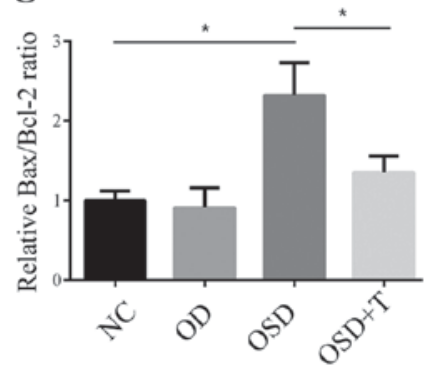

H

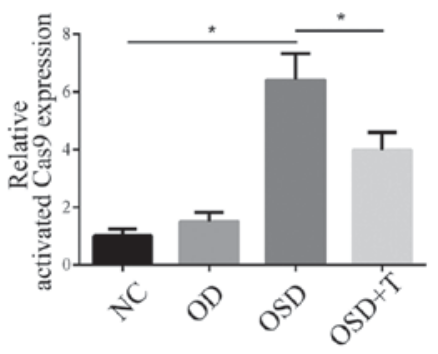

Figure 6. (A) Western blotting was performed to measure the activation of Cas3 and PARP. The expression of (B) Cas3, (C) activated Cas3, (D) PARP and (E) activated PARP was analyzed (F) Western blotting was performed to measure the expression of apoptosis-associated proteins. The expression of (G) Bax/Bcl-2 and (H) activated Cas9 was analyzed. ${ }^{*} \mathrm{P}<0.05$. Cas, caspase; PARP, poly ADP-ribose polymerase; Bax, B-cell lymphoma 2-associated X protein; Bcl-2, B-cell lymphoma 2 ; NC, negative control; OD, oxygen deprivation; OSD, oxygen and serum deprivation; T, transforming growth factor- 33 .

was significantly upregulated under OSD conditions, which indicates increased apoptosis (Fig. 6B and C). The results also demonstrated that caspase 3 activation was significantly decreased in the OSD+TGF- $\beta 3$ group compared with the OSD group (Fig. 6B and C). Bcl-2 and Bax are important mitochondria-mediated apoptotic proteins; Bax forms a complex with the anti-apoptotic protein Bcl-2 to balance its apoptotic effects and, as such, the ratio of $\mathrm{Bax} / \mathrm{Bcl}-2$ determines whether cells will undergo apoptosis (16). Caspase 9 is an important protein in the mitochondrial apoptotic pathway (17). Western blotting results demonstrated that OSD conditions induced an increase in the Bax/Bcl-2 ratio and caspase 9 activation (Fig. 6) and both were significantly decreased with the addition of TGF- $\beta 3$ (Fig. 6F-H).

PARP has been reported to modulate various DNA damage response (DDR) processes to ensure genomic integrity and induce DNA repair or cell death (18). PARP is cleaved by activated caspase 3 , and so changes in activated PARP are often consistent with activate caspase 3 levels (19). The results of western blotting revealed that activated PARP was upregulated in the OSD+TGF- $\beta 3$ group compared with the OD and OSD groups (Fig. 6D and E). These results demonstrate that OSD conditions induce increased apoptosis and DDR in ASDCs compared with OD alone. The addition of TGF- $\beta 3$ is able to alleviate OSD-induced apoptosis, which may be achieved via enhancement of the PARP-associated DNA repairing effect. To further confirm this, PARP knockdown ADSCs were constructed via siRNA transfection (Fig. 7A). The results of western blotting and Annexin V/PI staining revealed that PARP knockdown significantly abolished the protective effect of TGF- $\beta 3$ in ADSCs under OSD conditions (Fig. 7B and C).

\section{Discussion}

The results of the present study revealed that OD alone does not induce apoptosis in ADSCs, whereas OSD does. It was also demonstrated that the addition of TGF- $\beta 3$ is able to attenuate OSD-induced apoptosis by enhancing activated PARP. A previous in vivo study revealed that a low partial pressure of oxygen is optimal for ADSCs (20), which explains why OD did not induce apoptosis in the present study. TGF- $\beta 3$ is a member of the TGF family, which mainly functions through the Smad pathway (21). A previous study reported that TGF- $\beta 3$ is an important initiator of chondrocyte lineage differentiation (22). It has also been demonstrated that TGF- $\beta 3$ is associated with DNA mismatch repair genes (23). During embryonic 
A

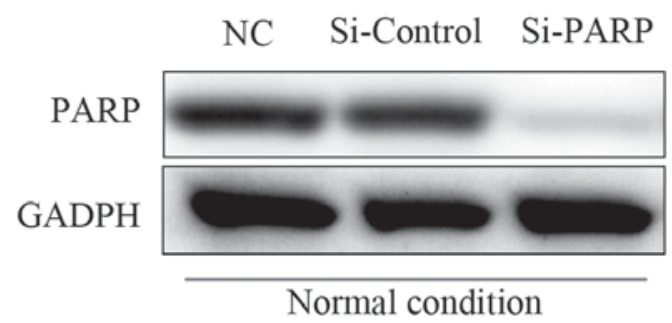

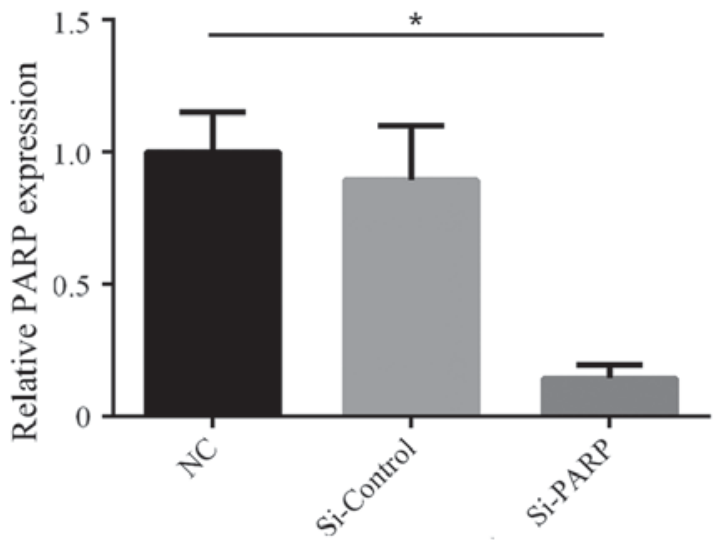
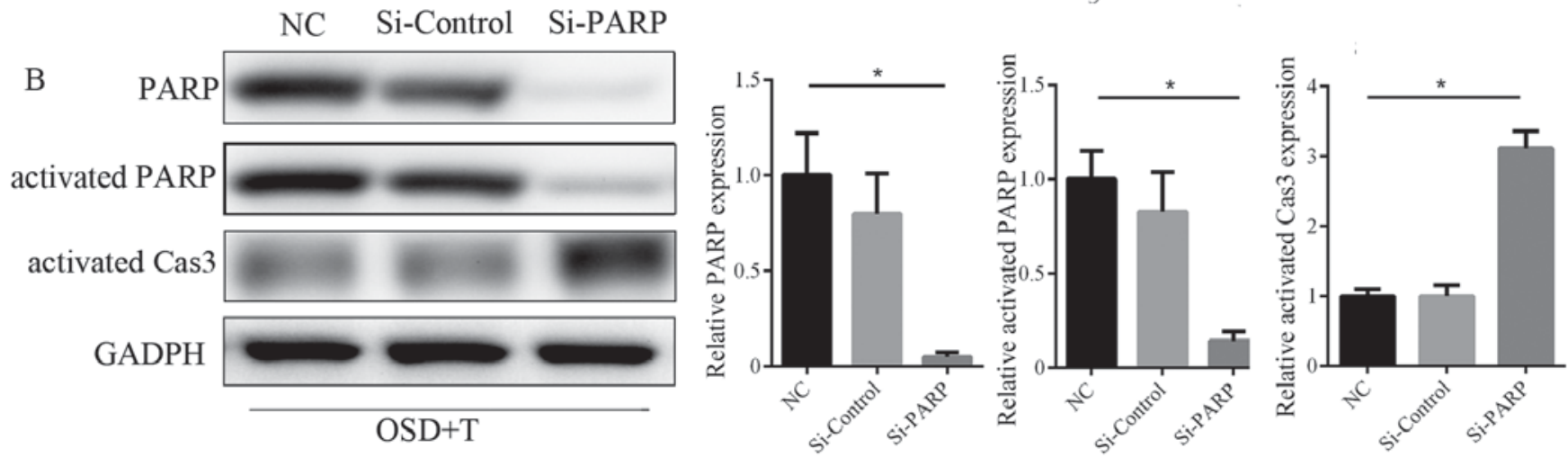

$\mathrm{C}$
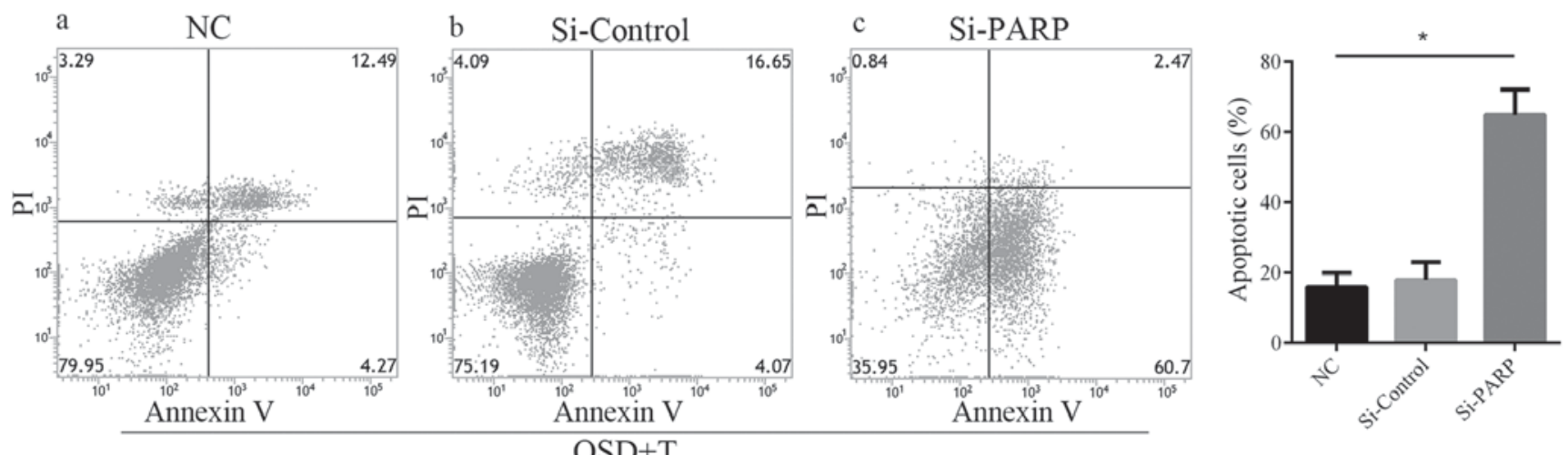

Figure 7. (A) Western blotting was performed to assess PARP expression in ADSCs transfected with si-PARP or si-Control. (B) Western blotting was performed to measure levels of PARP, activated PARP and activated Cas3 in transfected ADSCs cultured under OSD+T conditions. (C) Annexin V-PI staining was performed to assess apoptosis in ADSCs transfected with si-PARP or si-Control and cultured under OSD+T conditions. " $\mathrm{P}<0.05$. NC, negative control; ADSC, adipose-derived stem cells; PARP, poly ADP-ribose polymerase; si, small interfering; PI, propidium iodide; OSD, oxygen and serum deprivation; $\mathrm{T}$, transforming growth factor- $\beta 3$.

development, TGF- $\beta 3$ functions as a pro-apoptotic cytokine in some cells, including finger puff cells and is associated with palatogenesis $(23,24)$. TGF- $\beta 3$ also serves an anti-apoptotic role in the repair of tissue damage (25). TGF- $\beta 3$ is able to alleviate intestinal epithelial radiation damage and oral mucosal epithelial scratches, as well as increasing the survival rates of mice subjected to radiation injury (12).

In the present study, the addition of exogenous TGF- $\beta 3$ in OSD-treated ADSCs resulted in a decrease in apoptosis. Annexin-V/PI flow cytometric analysis revealed that the number of cells in the early stages of apoptosis increased in the OSD+TGF- $\beta 3$ group compared with OSD alone, however the total number of cells in the late stages of apoptosis was decreased. These results suggest that TGF- $\beta 3$ may inhibit apoptosis in
OSD-treated ADSCs, preventing ADSCs from entering the later stages of apoptosis. This result was further confirmed by a TUNEL array. The addition of exogenous TGF- $\beta 3$ significantly reduced the number TUNEL-positive cells, which represented a decrease of the programmed induction of DNA double strand breaks. It can then be hypothesized that when ADSCs are used as seed cells for cartilage tissue engineering, additional TGF- $\beta 3$ supplement may be necessary to alleviate OSD-associated cell death.

The results of western blotting revealed that the anti-apoptotic effects of TGF- $\beta 3$ may be achieved via the activation of PARP-associated DNA repair. Caspase 3 activation was upregulated in the OSD group and downregulated in the OSD+TGF- $\beta 3$ group. PARP knockdown significantly 
counteracts the protective effect of TGF- $\beta 3$ in ADSCs under OSD conditions. Among the molecular functions attributed to PARPs, their role in the DDR has been widely documented (18). In particular, PARP is associated with several cellular processes that respond to DNA lesions, including DDR, DNA signaling and DNA repair (18).

The results of the present study provide an insight into the role of TGF- $\beta 3$ in ADSCs under OSD conditions and may be used to develop and improve ADSCs-associated stem cell therapy with the use of exogenous TGF- $\beta 3$. However, the present study is not without limitations. Tissue engineering represents an attractive strategy for regenerative medicine, but cell survival following transplantation remains poor due to a combination of mechanical, metabolism and host factors, limiting the efficacy of stem cell therapy (26). OSD was used in the present study to mimic in vivo hypoxia based on previous studies (27-29). However, other factors, including glucose metabolism disorder and lactic acid accumulation, may also have an impact on cell survival in vivo (30) and were not considered in he present study. Future research should focus on the effect of TGF- $\beta 3$ on ADSCs in vivo under ischemic condition.

\section{Acknowledgements}

Not applicable.

\section{Funding}

The present study was supported by the Science and Technology Planning Project of Guangdong Province, China (grant no. 2012B061700104), the Key Laboratory of Malignant Tumor Molecular Mechanism and Translational Medicine of Guangzhou Bureau of Science and Information Technology [grant no. (2013) 163] and the Key Laboratory of Malignant Tumor Gene Regulation and Target Therapy of Guangdong Higher Education Institutes (grant no. KLB09001).

\section{Availability of data and materials}

The datasets used and analyzed during the current study are available from the corresponding author on reasonable request.

\section{Authors' contributions}

FW and HY cultured the cells, performed the experiments and were major contributors in writing the manuscript. JL and YX analyzed and interpreted the data. HZ and HX participated in the design of the study and analysis of data, and agree to be accountable for all aspects of work in ensuring that questions related to the accuracy of the study. ML and YZ designed the experiments, were involved in revising the manuscript for important intellectual content and participated in the analysis and interpretation of data. SC designed and directed the experiments.

\section{Ethics approval and consent to participate}

The study was approved by the Ethics Committee of Sun Yat-sen Memorial Hospital (Guangzhou, China).

\section{Consent for publication}

Not applicable.

\section{Competing interests}

The authors declare that they have no competing interests.

\section{References}

1. Hunziker EB: Articular cartilage repair: Basic science and clinical progress. A review of the current status and prospects. Osteoarthritis Cartilage 10: 432-463, 2002.

2. Rodriguez-Merchan EC: Regeneration of articular cartilage of the knee. Rheumatol Int 33: 837-845, 2013.

3. Shimomura K, Ando W, Fujie H, Hart DA, Yoshikawa H and Nakamura N: Scaffold-free tissue engineering for injured joint surface restoration. J Exp Orthop 5: 2, 2018.

4. Sterodimas A, de Faria J, Nicaretta B and Pitanguy I: Tissue engineering with adipose-derived stem cells (ADSCs): Current and future applications. J Plast Reconstr Aesthet Surg 63: 1886-1892, 2010.

5. Zheng DY, Dan Y, Yang SH, Liu GH, Shao ZW, Yang C, Xiao BJ, Liu X, Wu S, Zhang T and Chu PK: Controlled chondrogenesis from adipose-derived stem cells by recombinant transforming growth factor- $\beta 3$ fusion protein in peptide scaffolds. Acta Biomater 11: 191-203, 2015.

6. Cui Z, Shen L, Lin Y, Wang S, Zheng D and Tan Q: Inhibition of oxygen-glucose deprivation-induced apoptosis of human adipose-derived stem cells by genetic modification with antiapoptotic protein bcl-2. Aesthetic Plast Surg 38: 779-787, 2014.

7. Zhu S, Chen P, Wu Y, Xiong S, Sun H, Xia Q, Shi L, Liu H and Ouyang HW: Programmed application of transforming growth factor $\beta 3$ and rac1 inhibitor NSC23766 committed hyaline cartilage differentiation of adipose-derived stem cells for osteochondral defect repair. Stem Cells Transl Med 3: 1242-1251, 2014

8. Laverty HG, Wakefield LM, Occleston NL, O'Kane S and Ferguson MW: TGF-beta3 and cancer: A review. Cytokine Growth Factor Rev 20: 305-317, 2009.

9. Shen B, Wei A, Tao H, Diwan AD and Ma DD: BMP-2 enhances TGF-beta3-mediated chondrogenic differentiation of human bone marrow multipotent mesenchymal stromal cells in alginate bead culture. Tissue Eng Part A 15: 1311-1320, 2009.

10. Dünker N, Schmitt K and Krieglstein K: TGF-beta is required for programmed cell death in interdigital webs of the developing mouse limb. Mech Dev 113: 111-120, 2002.

11. Fogli M, Carlo-Stella C, Curti A, Ratta M, Tazzari PL, Ragazzi E, Colla S, Santucci AM, Tura S and Lemoli RM: Transforming growth factor beta3 inhibits chronic myelogenous leukemia hematopoiesis by inducing Fas-independent apoptosis. Exp Hematol 28: 775-783, 2000.

12. Booth D and Potten CS: Protection against mucosal injury by growth factors and cytokines. J Natl Cancer Inst Monogr: 16-20, 2001.

13. Mylotte LA, Duffy AM, Murphy M, O'Brien T, Samali A, Barry F and Szegezdi E: Metabolic flexibility permits mesenchymal stem cell survival in an ischemic environment. Stem Cells 26: 1325-1336, 2008.

14. Wang DW, Fermor B, Gimble JM, Awad HA and Guilak F: Influence of oxygen on the proliferation and metabolism of adipose derived adult stem cells. J Cell Physiol 204: 184-191, 2005.

15. D'Amelio M, Cavallucci V and Cecconi F: Neuronal caspase-3 signaling: Not only cell death. Cell Death Differ 17: 1104-1114, 2010.

16. Sharifi S, Barar J, Hejazi MS and Samadi N: Roles of the Bcl-2/Bax ratio, caspase- 8 and 9 in resistance of breast cancer cells to paclitaxel. Asian Pac J Cancer Prev 15: 8617-8622, 2014.

17. McIlwain DR, Berger T and Mak TW: Caspase functions in cell death and disease. Cold Spring Harb Perspect Biol 5: a008656, 2013.

18. Sousa FG, Matuo R, Soares DG, Escargueil AE, Henriques JA, Larsen AK and Saffi J: PARPs and the DNA damage response. Carcinogenesis 33: 1433-1440, 2012. 
19. Yang LJ, Chen Y,He J, Yi S, Wen L, Zhao S and Cui GH: Effects of gambogic acid on the activation of caspase-3 and downregulation of SIRT1 in RPMI-8226 multiple myeloma cells via the accumulation of ROS. Oncol Lett 3: 1159-1165, 2012.

20. Yue Y, Zhang P, Liu D, Yang JF, Nie C and Yang D: Hypoxia preconditioning enhances the viability of ADSCs to increase the survival rate of ischemic skin flaps in rats. Aesthetic Plast Surg 37: 159-170, 2013.

21. Zhang J, Zhang X, Xie F, Zhang Z, van Dam H, Zhang L and Zhou F: The regulation of TGF- $\beta /$ SMAD signaling by protein deubiquitination. Protein Cell 5: 503-517, 2014.

22. Bian L, Zhai DY, Tous E, Rai R, Mauck RL and Burdick JA: Enhanced MSC chondrogenesis following delivery of TGF- $\beta 3$ from alginate microspheres within hyaluronic acid hydrogels in vitro and in vivo. Biomaterials 32: 6425-6434, 2011.

23. He F, Xiong W, Wang Y, Li L, Liu C, Yamagami T, Taketo MM, Zhou $\mathrm{C}$ and Chen Y: Epithelial Wnt/ $\beta$-catenin signaling regulates palatal shelf fusion through regulation of Tgf $\beta 3$ expression. Dev Biol 350: 511-519, 2011.

24. Dunker N, Schmitt K and Krieglstein K: TGF-beta is required for programmed cell death in interdigital webs of the developing mouse limb. Mech Dev 113: 111-120, 2002.

25. Wu BX, Li A, Lei L, Kaneko S, Wallace C, Li X and Li Z Glycoprotein A repetitions predominant (GARP) positively regulates transforming growth factor (TGF) $\beta 3$ and is essential for mouse palatogenesis. J Biol Chem 292: 18091-18097, 2017.
26. Haleem AM and Chu CR: Advances in tissue engineering techniques for articular cartilage repair. Oper Tech Orthop 20:76-89, 2010.

27. Deng J, Han Y, Yan C, Tian X, Tao J, Kang J and Li S: Overexpressing cellular repressor of E1A-stimulated genes protects mesenchymal stem cells against hypoxia- and serum deprivation-induced apoptosis by activation of PI3K/Akt. Apoptosis 15: 463-473, 2010.

28. Zhu W, Chen J, Cong X, Hu S and Chen X: Hypoxia and serum deprivation-induced apoptosis in mesenchymal stem cells. Stem Cells 24: 416-425, 2006.

29. Hou M, Liu J, Liu F, Liu K and Yu B: C1q tumor necrosis factor-related protein-3 protects mesenchymal stem cells against hypoxia- and serum deprivation-induced apoptosis through the phosphoinositide 3-kinase/Akt pathway. Int J Mol Med 33: 97-1044, 2014

30. Deschepper M, Oudina K, David B, Myrtil V, Collet C, Bensidhoum M, Logeart-Avramoglou D and Petite H: Survival and function of mesenchymal stem cells (MSCs) depend on glucose to overcome exposure to long-term, severe and continuous hypoxia. J Cell Mol Med 15: 1505-1514, 2011. Attribution 4.0 International (CC BY-NC 4.0) License 